\title{
ASTROPHYSICS AND COSMOLOGY
}

\section{COMPARATIVE ANALYSIS OF STANDARD $\Lambda$ CDM AND $\Lambda$ CS MODELS}

\author{
V.E. KUZMICHEV, V.V. KUZMICHEV
}

PACS 98.80.Qc; 98.80.Cq;

Bogolyubov Institute for Theoretical Physics, Nat. Acad. of Sci. of Ukraine

(c) 2012

(14b, Metrolohichna Str., Kyiv 03680, Ukraine; e-mail: vkuzmichev@bitp. kiev. ua)

We draw a comparison of time-dependent cosmological parameters calculated in the standard $\Lambda \mathrm{CDM}$ model with those of the model of a homogeneous and isotropic Universe with non-zero cosmological constant filled with a perfect gas of low-velocity cosmic strings ( $\Lambda \mathrm{CS}$ model). It is shown that pressure-free matter can obtain the properties of a gas of low-velocity cosmic strings in the epoch, when the global geometry and the total amount of matter in the Universe as a whole obey an additional constraint. This constraint follows from the quantum geometrodynamical approach in the semiclassical approximation. In terms of general relativity, its effective contribution to the field equations can be linked to the time evolution of the equation of state of matter caused by the processes of redistribution of the energy between matter components. In the present article, the exact solutions of the Einstein equations for the $\Lambda$ CS model are found. It is demonstrated that this model is equivalent to the open de Sitter model. After the scale transformation of the time variable of the $\Lambda$ CS model, the standard $\Lambda \mathrm{CDM}$ and $\Lambda \mathrm{CS}$ models provide the equivalent descriptions of cosmological parameters as functions of time at equal values of the cosmological constant. The exception is the behavior of the deceleration parameter in the early Universe.

\section{Introduction}

The standard $\Lambda$ CDM model (see, e.g., reviews $[1,2]$ ) gives the satisfactory description of the most of the present cosmological data under the assumption of the existence of an antigravitating medium named dark energy as the largest constituent of mass-energy in the Universe. At the same time, it is believed that a high level of fine-tuning is required in this model. Even if the smallness of the cosmological constant and the "coincidence problem" (the almost equal contributions of matter and dark energy to the total energy budget of the Universe at the present era) are not problems in themselves [3], nevertheless it should not be ignored that there were some indications that specific cosmological observations differed from the predictions of the $\Lambda$ CDM model at a statistically significant level [4].

The $\Lambda$ CDM model based on general relativity allows extensions by incorporating new elements in its scheme. For example, one of such possibilities is the introduction of the quintessence field, which changes over time and is described by some dynamic equation, instead of the cosmological constant. Another opportunity may be the model, in which, alternatively, the gravitating matter component undergoes a modification, regardless of the vacuum component of the energy density being constant or varying with time. Such a modification may be made, by relying on the fundamental physical laws, which concern the properties of matter.

In the FRW cosmology, the time evolution of the energy density $\rho(t)$ is determined by the equation

$\dot{\rho}+3 \frac{\dot{R}}{R}(\rho+p)=0$,

where $R(t)$ is the cosmological scale factor, $p$ is the isotropic pressure, and the dot designates the derivative with respect to the proper time $t$. For the equation of state in the form $p=w \rho$, the solution of this equation vanishing at infinity can be written as $\rho=\mu R^{-3(1+w)}$, where $w$ and $\mu$ are constants. Introducing the effective mass $M_{\text {eff }}$ contained in the volume $\sim R^{3}$ by the relation $M_{\text {eff }} \sim \rho R^{3}$, we have $M_{\text {eff }} \sim \mu R^{-3 w}$. For the special case $w=0$, it gives $M_{\text {eff }} \sim \mu=$ const, which corresponds to pressure-free matter (dust). For $w=-\frac{1}{3}$, the effective mass is proportional to the scale factor, $M_{\text {eff }} \sim \mu R$. In this case, the energy density $\rho \sim R^{-2}$, and it describes the so-called K-matter [5]. The matter with such energy 
density and equation of state can be interpreted as a perfect gas of low-velocity cosmic strings [6].

In this paper, we study the model of a homogeneous and isotropic Universe with non-zero cosmological constant filled with a perfect gas of low-velocity cosmic strings. Throughout the paper, we will refer to this model as the $\Lambda$ CS model. It is shown that pressure-free matter can obtain the properties of a gas of low-velocity cosmic strings, if, in addition to the field equations, there exists a complementary constraint between the global geometry and the total amount of matter in the Universe as a whole. We show that this constraint between the cosmological parameters, which takes the form of the geometry-mass relation, can be obtained in the quantum geometrodynamical approach. In terms of general relativity, its effective contribution to the field equations can be linked to the time evolution of the equation of state of matter caused by the processes of redistribution of the energy between matter components. This is demonstrated in the model, in which a two-component perfect fluid serves as a surrogate for matter in the Universe.

We found the exact solutions of the Einstein equations for the $\Lambda \mathrm{CS}$ model. It is demonstrated that this model is equivalent to the open de Sitter model. In the limit of zero cosmological constant, the corresponding Universe evolves as a Milne Universe characterized by the linear dependence of the scale factor on time. But, in contrast to it, such a Universe contains matter with nonzero energy density in the form of a perfect gas of low-velocity cosmic strings. The Whitrow-Randall equation [7], which establishes the invariance of the dimensionless product $G \rho t^{2}$, is re-derived. We make a comparison of the standard $\Lambda \mathrm{CDM}$ and $\Lambda \mathrm{CS}$ models. It turns out that, after the scale transformation $t \rightarrow \frac{3}{2} t$ of the time variable of the $\Lambda \mathrm{CS}$ model, these models provide the equivalent descriptions of cosmological parameters as functions of time at equal values of the cosmological constant. The exception is the behavior of the deceleration parameter in the early Universe. But, for the present day and in the future, it would be more difficult to recognize whether one is dealing with the $\Lambda$ CDM- or $\Lambda$ CS-Universe.

\section{Quantum Roots of the Geometry-Mass Relation}

It is well known that quantum theory adequately describes properties of various physical systems. Its universal validity demands that the Universe as a whole must obey quantum laws as well, so that quantum effects are important at least in the early era. Since quantum effects are not a priori restricted to certain scales, one should not conclude in advance that they cannot have any impact on the processes on scales larger than the Planckian one (more detailed arguments can be found, e.g., in Refs. [8]).

Quantum theory for a homogeneous and isotropic Universe can be constructed on the basis of a Hamiltonian formalism with the use of a material reference system as a dynamical system $[9,10]$. Defining the time parameter or the "clock" variable, it is possible to pass from the Wheeler-DeWitt equation to the Schrödinger-type equation. The similar equations containing a time variable defined by means of the coordinate condition were considered by a number of authors under the quantization of the FRW Universe (see, e.g., Refs. [11]). Using the Schrödinger-type equation, one can obtain the equations of motion for the expectation values of a scale factor and its conjugate momenta. These equations pass into the equations of general relativity, when the dispersion around the expectation values for a scale factor, matter fields, and their conjugate momenta can be neglected.

Such a quantum theory predicts that the following relation must hold for the expectation value of the scale factor $R$ in the state $|M\rangle$, which describes the Universe with a definite total amount of mass $M$ much larger than the Planck mass, $M \gg M_{\mathrm{P}}$ :

$$
\frac{\langle M|R| M\rangle}{\langle M \mid M\rangle}=G M
$$

(in units $c=1$; for details, see Refs. [10]), where $G$ is the Newtonian gravitational constant.

Equation (2) determines the mass $M$ through the expectation value of the scale factor $R$ at every instant of time. The state vector of the isotropic Universe is a superposition of all possible $|M\rangle$ characterizing the states which are not orthogonal between themselves, so that the inner product $\left\langle M_{1} \mid M_{2}\right\rangle \neq 0$, and the Universe can transit spontaneously from the state with mass $M_{1}$ and radius $R_{1}=\left\langle M_{1}|R| M_{1}\right\rangle /\left\langle M_{1} \mid M_{1}\right\rangle$ to the state with mass $M_{2} \neq M_{1}$ and radius $R_{2}=\left\langle M_{2}|R| M_{2}\right\rangle /\left\langle M_{2} \mid M_{2}\right\rangle$ with the nonzero probability $P(1 \rightarrow 2)=\left|\left\langle M_{1} \mid M_{2}\right\rangle\right|^{2}$. For example, the probability of the transition of the Universe from the ground state (with respect to the gravitational field) to any other state obeys the Poisson distribution with the mean number of occurrences $n=\frac{1}{2}\left(M_{2}-M_{1}\right)^{2}$ (for more details, see Refs. [10]). Thus, $R_{1} \rightarrow R_{2}$, when $M_{1} \rightarrow M_{2}$.

In the classical limit, it appears to be possible to pass from the expectation value of $R$ to the classical value of the scale factor $R(t)$, which evolves in time in accordance 
with the Einstein equations for the FRW Universe:

$\dot{R}^{2}=\frac{8 \pi G}{3} \rho R^{2}+\frac{\Lambda}{3} R^{2}-k$,

$\ddot{R}=-\frac{4 \pi G}{3}(\rho+3 p) R+\frac{\Lambda}{3} R$

Here,

$\rho=\frac{M}{(4 \pi / 3) R^{3}}$

is the energy density of matter with the total mass $M$ in the equivalent flat-space volume $(4 \pi / 3) R^{3}$, which includes both the mass of substance and the mass equivalent of radiation energy, $\Lambda$ is the cosmological constant,

$p=-\rho-\frac{R}{3} \frac{d \rho}{d R}$

is the isotropic pressure, and $k=+1,0,-1$ for the spatially closed, flat, or open models. In the semiclassical limit, relation (2) takes the form

$R=G M$

It gives an additional constraint between the global geometry and the total amount of matter in the Universe as a whole. The geometry-mass relation (6) connects the values of $M$ and $R$ taken at the same instant of time. It is valid for the present-day Universe. Really, the radius of its observed part is estimated as $R_{0} \sim 10^{28} \mathrm{~cm}$, the mass-energy is $M_{0} \sim 10^{56} \mathrm{~g}$, and the mean energy density equals $\rho_{0} \sim 10^{-29} \mathrm{~g} \mathrm{~cm}^{-3}$. This means that, nowadays, $\rho_{0} \sim 3\left(4 \pi G R_{0}^{2}\right)^{-1}$. Then, from the definition of energy density $\rho_{0}=3 M_{0}\left(4 \pi R_{0}^{3}\right)^{-1}$, it follows that the relation $R_{0} \sim G M_{0}$ must hold. It is notable that, for the values $R=L_{P}, M=M_{P}$, where $L_{P}$ is the Planck length, Eq. (6) reduces to the identity.

The physical meaning of relation (6) will be discussed in Section 5. Here, we remark only that since it is valid at least at late times $t \sim t_{0}$, where $t_{0}$ is the age of the Universe, the theory which includes the geometry-mass relation (6) can be used for the description of the evolution of the Universe on the interval $t=t_{0} \mp \Delta t, \Delta t / t_{0} \ll 1$.

\section{3. $\Lambda$ CS Model}

If one supposes that the values of $R$ and $M$ in Eq. (6) are constant, then the FRW Universe described by Eqs. (3) transforms into the static Einstein Universe [12]. Let us consider the more general case, by assuming that relation
(6) is valid for some time interval and can be regarded as a constraint added to the classical field equations (3). Then the energy density of matter (4) takes the form of the energy density of a gas of low-velocity cosmic strings or K-matter $[5,6]$ with the corresponding equation of state,

$\rho=\frac{3}{G} \frac{1}{4 \pi R^{2}}, \quad p=-\frac{1}{3} \rho$.

However, in this approach, this does not mean that the Universe is string-dominated in the usual sense. The energy density and the pressure in the form (7) arise as the effect of an additional constraint between the global geometry and the total amount of matter in the Universe as a whole.

Let us consider the model of the Universe with cosmological constant $\Lambda_{s}$ and the matter density and the pressure as those in Eq. (7). The field equations are reduced to the form

$\dot{R}^{2}=\frac{\Lambda_{s}}{3} R^{2}+(2-k), \quad \ddot{R}=\frac{\Lambda_{s}}{3} R$.

Their solution is

$R_{s}(t)=\sqrt{\frac{6}{\Lambda_{s}}\left(1-\frac{1}{2} k\right)} \sinh \left(\sqrt{\frac{\Lambda_{s}}{3}} t\right), R_{s}(0)=0$.

Here and below, the subscript $s$ refers to the $\Lambda$ CS model. Expansion of this solution for small $\left|\frac{\Lambda_{s}}{3} t^{2}\right|$ yields

$R_{s}(t)=\sqrt{2-k} t\left[1+\frac{1}{6}\left(\frac{\Lambda_{s}}{3} t\right)^{2}+\ldots\right]$

The Hubble expansion rate does not depend on the type of a spatial curvature (the value of $k$ ) and is described by the expression

$H_{s}(t)=\frac{\dot{R_{s}}}{R_{s}}=\sqrt{\frac{\Lambda_{s}}{3}} \operatorname{coth}\left(\sqrt{\frac{\Lambda_{s}}{3}} t\right)$.

The expansion of $H_{s}(t)$ in the same limit as above has a form

$H_{s} t=1+\frac{1}{3}\left(\frac{\Lambda_{s}}{3}\right) t^{2}-\frac{1}{45}\left(\frac{\Lambda_{s}}{3}\right)^{2} t^{4}+\ldots$.

In the general case, the Hubble expansion rate $H$ is a function of time, and the corresponding critical energy density is $\rho_{\mathrm{cr}}(t)=\frac{3 H^{2}(t)}{8 \pi G}$. Then the time variation of the vacuum energy density parameter $\Omega_{\Lambda}(t) \equiv \frac{\rho_{\Lambda}}{\rho_{\mathrm{cr}}(t)}$, where $\rho_{\Lambda}=\frac{\Lambda}{8 \pi G}$, is given by

$\Omega_{\Lambda s}(t)=\tanh ^{2}\left(\sqrt{\frac{\Lambda_{s}}{3}} t\right)$ 
for the $\Lambda$ CS model. The matter energy density parameter $\Omega_{M}(t)=\frac{\rho}{\rho_{\mathrm{cr}}(t)}$ for a spatially flat Universe filled with a gas of low-velocity cosmic strings is equal to

$\Omega_{M s}(t)=\cosh ^{-2}\left(\sqrt{\frac{\Lambda_{s}}{3}} t\right)$.

Setting $\Lambda_{s}=3 \Omega_{\Lambda 1} H_{s}^{2}\left(t_{1}\right)$, where $t_{1}$ is some fixed instant of time, and $\Omega_{\Lambda 1}$ is the vacuum energy density parameter $\Omega_{\Lambda s}$ at $t=t_{1}$, we find

$H_{s}\left(t_{1}\right) t_{1}=\frac{1}{\sqrt{\Omega_{\Lambda 1}}} \operatorname{arctanh} \sqrt{\Omega_{\Lambda 1}}$.

The deceleration parameter $q(t)=-\frac{\ddot{R}}{R H^{2}(t)}$ is equal to

$q_{s}(t)=-\Omega_{\Lambda s}(t)$

At an instant of time $t=t_{1}$, we obtain $q_{s}\left(t_{1}\right)=-\Omega_{\Lambda 1}$.

If $\Lambda_{s} \neq 0$, the expressions for the scale factor (9) and the Hubble expansion rate (11) are equivalent to the respective expressions in the de Sitter model of the Universe with $k=-1$.

In the limiting case $\Lambda_{s}=0$, it appears that

$R_{s}(t)=\sqrt{2-k} t, \quad H_{s} t=1$.

In the model, where the scale factor depends on time linearly, the age of the Universe and the Hubble expansion rate depend on the redshift $z$ according to the simple laws

$t(z)=\frac{1}{(1+z) H_{s}(0)}, \quad H_{s}(z)=H_{s}(0)(1+z)$.

Taking the present expansion rate measured by observations with a Hubble Space Telescope, $H(0)=73.8 \pm 2.4$ $\mathrm{km} \mathrm{s}^{-1} \mathrm{Mpc}^{-1}$ [13], as $H_{s}(0)$, we find that the age of the Universe appears to be equal $t(0)=13.26 \pm 0.43 \mathrm{Gyr}$. This value does not differ drastically from the value predicted by the WMAP 7-year data [14] for the $\Lambda$ CDM model, and it lies within the expected limit of 12 to 14 Gyr.

Solution (17) formally coincides with the solution of the Milne model of an open Universe $(k=-1), R(t) \sim t$. But, in contrast to the Milne model, where the energy density of matter vanishes, $\rho=0$, the energy density of matter is nonzero,

$\rho=\frac{3 H^{2}}{4 \pi G(2-k)}$, in the case under consideration. For a spatially flat Universe with zero cosmological constant, this density equals to the critical density, $\rho=\rho_{\mathrm{cr}}$. For a spatially closed Universe filled with a gas of low-velocity cosmic strings, the density is $\rho=2 \rho_{\text {cr }}$, and we have $\rho=\frac{2}{3} \rho_{\text {cr }}$ for a spatially open Universe.

It should be noted that the Milne model cannot be correct near the point of the initial cosmological singularity, $t=0$, since the energy density of matter tends to infinity in this limit, and gravity cannot be neglected. There was an attempt to preserve the linear dependence of a scale factor on time and to get rid of this shortcoming of the Milne model by consideration of the model (called the "Dirac-Milne" Universe by analogy with the sea of positive and negative energy states proposed by Dirac), in which the Universe contains the equal amounts of matter with positive and negative gravitational masses [15].

Equation (19) can be rewritten in the WhitrowRandall form [7],

$G \rho t^{2}=\frac{3}{4 \pi} \frac{1}{n}$,

which shows that $G \rho t^{2}$ is an invariant determined by the parameter $n=2-k$ characterizing the geometry of the Universe.

Introducing a dimensionless parameter $K$ like the model of K-matter,

$K \equiv \frac{8 \pi G}{3} \rho R^{2}$,

and using (7), one finds that $K=2$. This value agrees with the observational constraints on the parameter $K$ obtained by Kolb [5] and Gott and Rees [16].

\section{Comparison with the Standard Cosmological Model}

The relations obtained in Section 3 for the cosmological parameters of the $\Lambda \mathrm{CS}$ model of the Universe can be compared with the corresponding expressions for the standard cosmological model. First of all, it is helpful to rewrite the equation for $\dot{R}$ in Eq. (3) in the form

$\dot{R}^{2}=\frac{\Lambda}{3} R^{2}+(2-k)+\zeta(R)$,

where the function $\zeta(R)$ is defined as

$\zeta(R)=\frac{2}{R}(G M-R)$

Comparing Eqs. (8) and (22), we find that the function $\zeta(R)$ describes the difference between the model which 
takes the geometry-mass relation (6) into account and the model without regard for it.

For a spatially flat Universe $(k=0)$ filled with pressure-free matter ( $p=0, M=$ const), the solution of Eq. (22) reads (cf. Ref. [17])

$$
R(t)=\left(\frac{6}{\Lambda} G M\right)^{1 / 3} \sinh ^{2 / 3}\left(\frac{3}{2} \sqrt{\frac{\Lambda}{3}} t\right), R(0)=0 .
$$

From Eqs. (9) and (24), it follows that the scale factors of both models can be connected by the relation

$$
R(t)=\left(\frac{G M}{1-\frac{1}{2} k}\right)^{1 / 3} R_{s}^{2 / 3}\left(\frac{3}{2} t\right) \quad \text { at } \quad \Lambda=\Lambda_{s} .
$$

This expression establishes the rule of recalculation of the scale factor from the $\Lambda$ CS model with arbitrary spatial curvature to the spatially flat standard model. This connection between two models becomes more clear if one considers relation (6) in its weaker form in solution (24), namely, by assuming that it is valid at some fixed instant of time $t_{0}$ only. As we have already mentioned above, this relation is realized in the present-day Universe. Setting $R\left(t_{0}\right)=G M\left(t_{0}\right)$, where $M\left(t_{0}\right)$ is the mass of matter in the Universe with the "radius" $R\left(t_{0}\right)$, we have

$R\left(t_{0}\right)=\sqrt{\frac{6}{\Lambda}} \sinh \left(\frac{3}{2} \sqrt{\frac{\Lambda}{3}} t_{0}\right)$.

Then relation (25) at a fixed instant of time takes the form

$R\left(t_{0}\right)=\left(1-\frac{1}{2} k\right)^{-1 / 2} R_{s}\left(\frac{3}{2} t_{0}\right)$ at $\Lambda=\Lambda_{s}$.

The Hubble expansion rate in the standard model is

$H(t)=\frac{\dot{R}}{R}=\sqrt{\frac{\Lambda}{3}} \operatorname{coth}\left(\frac{3}{2} \sqrt{\frac{\Lambda}{3}} t\right)$.

Comparing Eq. (28) with Eq. (11), we find that the Hubble expansion rates calculated for both models are related between themselves by the simple relation

$H(t)=H_{s}\left(\frac{3}{2} t\right) \quad$ at $\quad \Lambda=\Lambda_{s}$.

The expansion of $H(t)$ for small $\left|\frac{\Lambda}{3} t^{2}\right|$ can be obtained from Eq. (12) by the substitution of $\frac{3}{2} t$ for $t$ and $\Lambda$ for $\Lambda_{s}$. This expansion of $H(t)$ reproduces the familiar expression $H=\frac{2}{3 t}$ for $\Lambda=0$ in contrast to $H_{s}=\frac{1}{t}$ for the $\Lambda$ CS model with $\Lambda_{s}=0$.
The Hubble expansion rates as functions of the dimensionless time parameter $t / t_{0}$, where $t_{0}$ is the age of the Universe in the $\Lambda$ CDM model, are plotted in Fig. 1. It is supposed that the cosmological constant in both models is the same. The WMAP 7-year data [14] for the present-day cosmological parameters are used. As we can see, the value of $H(t)$ at $t=t_{0}$ coincides with the value of $H_{s}(t)$ at $t=\frac{3}{2} t_{0}$.

Taking Eqs. (29), (13), and (14) into account, we find the relations

$\Omega_{\Lambda}(t)=\Omega_{\Lambda s}\left(\frac{3}{2} t\right)$

$\Omega_{M}(t)=\Omega_{M s}\left(\frac{3}{2} t\right)$ at $\Lambda=\Lambda_{s}$,

where $\Omega_{\Lambda}(t)$ and $\Omega_{M}(t)$ are the vacuum and matter energy density parameters of the standard model.

The energy density parameters of the vacuum and matter as functions of time are depicted in Fig. 2. It is assumed that the Universe is spatially flat. As for the Hubble parameter, the $\Lambda$ CS model reproduces the results of the standard model after the time transformation $t \rightarrow \frac{3}{2} t$.

Introducing the vacuum energy density parameter $\Omega_{\Lambda 0}$ at a fixed instant of time $t=t_{0}$ by the relation $\Lambda=$ $3 \Omega_{\Lambda 0} H^{2}\left(t_{0}\right)$, we get

$H\left(t_{0}\right) t_{0}=\frac{2}{3} \frac{1}{\sqrt{\Omega_{\Lambda 0}}} \operatorname{arctanh} \sqrt{\Omega_{\Lambda 0}}$.

From the comparison of Eq. (31) with Eq. (15), it follows that, at equal Hubble expansion rates according to Eq. (29) and equal contributions of the vacuum energy densities into the matter-energy budget of the Universe, $\Omega_{\Lambda 0}=\Omega_{\Lambda 1}$, the parameter $t_{1}=\frac{3}{2} t_{0}$. This means that if one defines $t_{0}$ and $t_{1}$ as the ages of the Universe in both models under consideration, then the age $t_{1}$ for the $\Lambda$ CS model will be 1.5 times greater than that for the standard model.

The last equation establishes the correspondence between the parameters $H\left(t_{0}\right), t_{0}$, and $\Omega_{\Lambda 0}$. Substituting the WMAP 7-year data [14] for the age of the Universe $t_{0}=13.75 \pm 0.13 \mathrm{Gyr}$, the Hubble parameter $H\left(t_{0}\right)=$ $71.0 \pm 2.5 \mathrm{~km} \mathrm{~s}^{-1} \mathrm{Mpc}^{-1}$, and the dark energy density parameter $\Omega_{\Lambda 0}=0.734 \pm 0.029$, which corresponds to the cosmological constant $\Lambda=(1.302 \pm 0.143) \times 10^{-56}$ $\mathrm{cm}^{-2}$, into Eq. (31), one finds a consistent result for our Universe: $H\left(t_{0}\right) t_{0}=0.998 \pm 0.045$.

The deceleration parameter

$q(t)=\frac{1}{2}\left[1-3 \tanh ^{2}\left(\frac{3}{2} \sqrt{\frac{\Lambda}{3}} t\right)\right]$. 


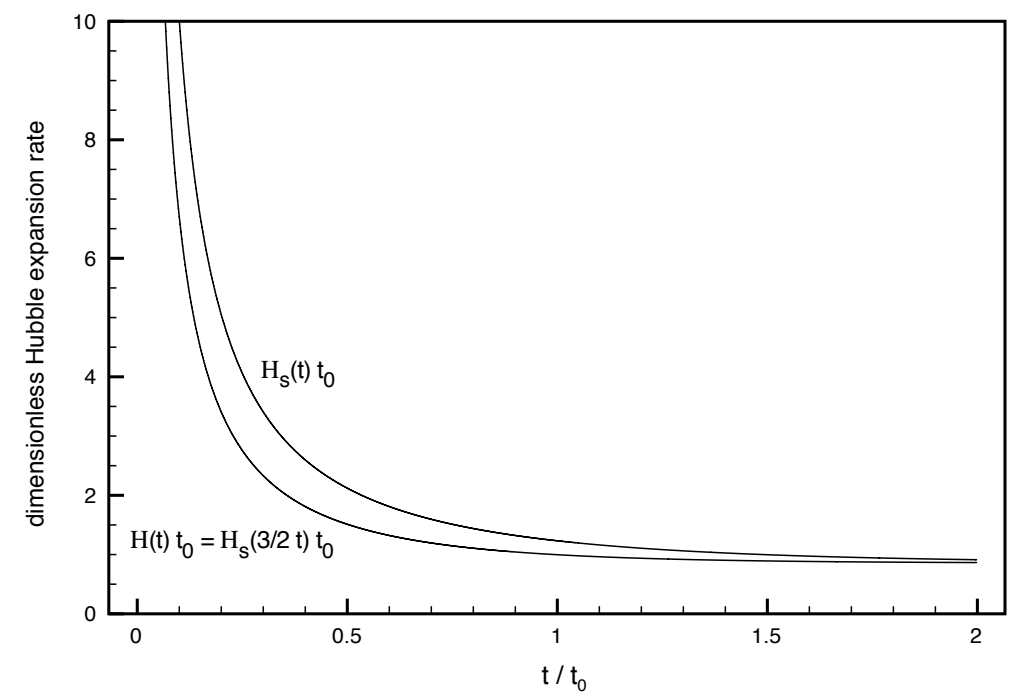

Fig. 1. Hubble expansion rates (11) and (28) as functions of time. The value of parameter $\sqrt{\frac{\Lambda}{3}} t_{0}=0.855$ following from the WMAP 7-year data [14] is used. It is assumed that $\Lambda=\Lambda_{s}$

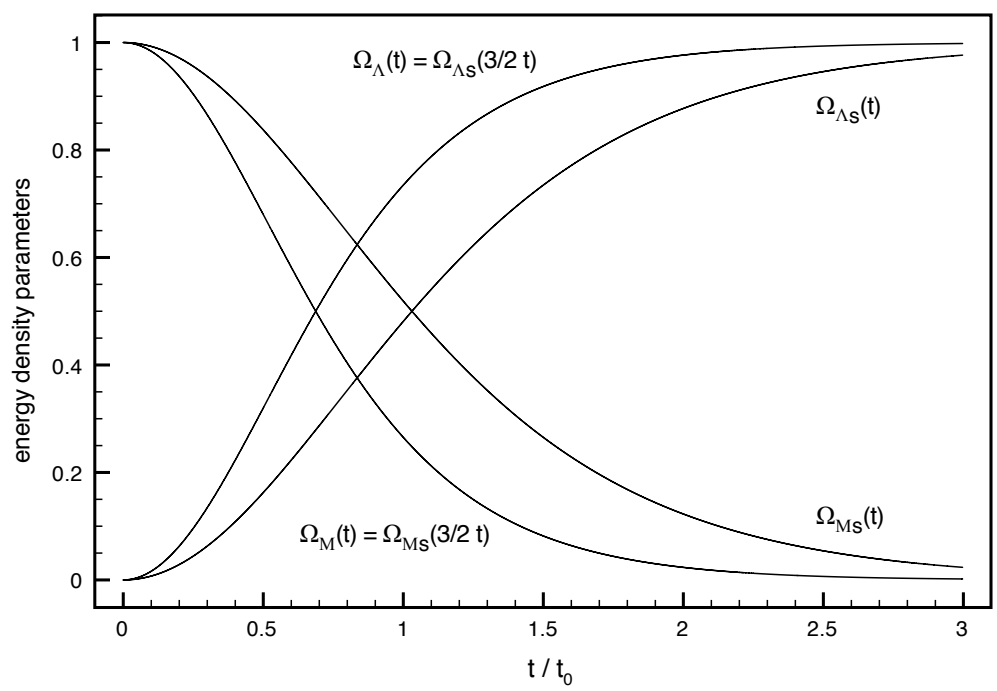

Fig. 2. Energy density parameters of the vacuum and matter (13), (14), and (30) as functions of time in units of $t_{0}$. The WMAP 7 -year data are used (see the caption of Fig. 1)

At an instant of time $t=t_{0}$, we have

$q\left(t_{0}\right)=\frac{1}{2}\left[1-3 \Omega_{\Lambda 0}\right]$

Comparing Eqs. (16) and (32), we find that both expressions for the deceleration parameter have the same limit as $t \rightarrow \infty, q(t) \rightarrow-1$, and $q_{s}(t) \rightarrow-1$, but they have different values at $t=0, q(0)=\frac{1}{2}$, and $q_{s}(0)=0$. From condition (29) valid at $\Lambda=\Lambda_{s}$, we have $\Omega_{\Lambda 0}=\Omega_{\Lambda 1}$, and expression (33) can be rewritten as

$q\left(t_{0}\right)=\frac{1}{2}\left[1+3 q_{s}\left(\frac{3}{2} t_{0}\right)\right]$.

In Fig. 3, we show the time dependence of the deceleration parameters $q(t)$ and $q_{s}(t)$ for the standard and $\Lambda \mathrm{CS}$ models. The function $q_{s}\left(\frac{3}{2} t\right)$ with the argument multiplied by $\frac{3}{2}$ is plotted for comparison. For $\frac{t}{t_{0}} \gtrsim 2.5$, the curves $q(t)$ and $q_{s}\left(\frac{3}{2} t\right)$ practically coincide. The both models predict the accelerating expansion of the Universe at $t=t_{0}$ and give the close values of the decelera- 
tion parameter, $q\left(t_{0}\right)=-0.601$ and $q_{s}\left(\frac{3}{2} t_{0}\right)=-0.734$. They lead to the same limit as $t \rightarrow \infty, q(t) \rightarrow-1$, and $q_{s}(t) \rightarrow-1$. But, in the region $\frac{t}{t_{0}}<1$, the behaviors of the functions differ drastically. The standard model predicts that, for $\frac{t}{t_{0}}<0.5$, the Universe decelerates. On the contrary, the $\Lambda \mathrm{CS}$ model describes the Universe with non-zero cosmological constant, which always accelerates and contains matter in the form of a perfect gas of lowvelocity cosmic strings.

The fact that the predictions concerning the deceleration parameter in both models differ for the time interval $0<t<\frac{1}{2} t_{0}$ is not surprising. The $\Lambda$ CS model in the form under consideration does not claim to provide a satisfactory description of the Universe at all times. This model is inapplicable to describe the expansion of the Universe at the times, when the geometry-mass relation (6) has no impact on the properties of matter.

At the same time, we can conclude that, for the present-day and future Universe, it would be more difficult to distinguish between the $\Lambda \mathrm{CDM}$ and $\Lambda \mathrm{CS}$ models. It looks like the Universe at some instant of time becomes dominated by matter in the form of a perfect gas of low-velocity cosmic strings. The reason for such a transformation of matter is different from that, which leads to the formation of macroscopic topological defects in the form of strings in the early Universe, where they are caused by phase transitions (see Ref. [18] and references therein). In the later Universe the energy density and the pressure in the form as for a perfect gas of low-velocity cosmic strings may arise as the effect of an additional constraint between the global geometry and the total amount of matter in the Universe as a whole.

Thus, the $\Lambda \mathrm{CDM}$ and $\Lambda \mathrm{CS}$ models lead to similar predictions on cosmological parameters, if the time variable of the $\Lambda$ CS model is subjected to the scale transformation $t \rightarrow \frac{3}{2} t$. After this transformation, the Hubble expansion rate and the energy density parameters of matter and vacuum components of the Universe, which are calculated for all instants of time, take equal values in both models.

\section{Discussion}

Let us consider the possible physical interpretation of the geometry-mass relation (6). First of all, we point out that the similar equality between the mass and the "radius" of the Universe was obtained by Whitrow and Randall [7] (see Eq. (20) for $k=0$ ). Such a relation is also valid for the Einstein Universe filled with the pressure-free matter (see, e.g., Ref. [12]) and for the steady-state cosmology [19].
Further on, it should be noted that relation (6) has a form of Sciama's inertial force law $M \sim G^{-1} R$, where $M$ and $R$ are the appropriate values of mass and radius of the observed part of the Universe [20,21]. Despite its simplified character, Sciama's linearized theory gives a specific mathematical relation between the parameters which characterize the energy density and the geometry of the Universe and corresponds to one of the realizations of Mach's principle [22-24].

If one assumes that Mach's principle is a fundamental law of the Nature, it must be implemented into the classical field equations. One viewpoint is that Einstein's field equations need not to be modified, while Mach's principle should be considered as an additional condition. Such an approach was chosen by Wheeler who proposed to understand Mach's principle as a selection rule (boundary condition) of the solutions of the field equations [25]. The Brans-Dicke theory uses another way, in which the field equations are generalized to become Machian [26, 27].

Since the scale factor $R$ obeys Eqs. (3), the mass $M$, generally speaking, must evolve in time. This means that if the gravitational constant $G$ and velocity of light $c$ are both constant, the mass of matter in the Universe must change proportionally to the scale factor, $M \sim R(t)$, at the time interval, where relation (6) holds.

In some cosmological models, the natural constant $G$ or $c$ is supposed to change with time. For example, according to Dirac's large number hypothesis, the Newtonian constant $G$ and the scale factor $R$ must depend on time as $G \sim t^{-1}$ and $R \sim t^{1 / 3}$ [28] or $G \sim t^{-1}$ and $R \sim t[29]$. Another example with varying $G$ is the Brans-Dicke theory, where this quantity is related to the average value of some dynamical scalar field $\phi$ coupled to the mass density $\rho$ of the Universe, $\langle\phi\rangle \approx G^{-1}$, where $\langle\phi\rangle \sim \rho R^{2}[26,30]$. The models with varying speed of light were also considered and applied to solve the horizon, flatness, cosmological constant, and other cosmological problems (see, e.g., Refs. [31]). On the other hand, there exist the observational and experimental bounds on the time variation of the fundamental constants (e.g., Ref. [32]).

The possible dependence of the mass $M$ on time can be considered within a fundamentally different approach, which deals with the matter creation processes in the context of the cosmological models [33]. But, currently, the models with the irreversible creation of matter do not rely on the sufficient observational evidence.

We shall use another approach which does not involve the theoretical schemes mentioned above in this section. Relation (6) follows from quantum theory in the semi- 


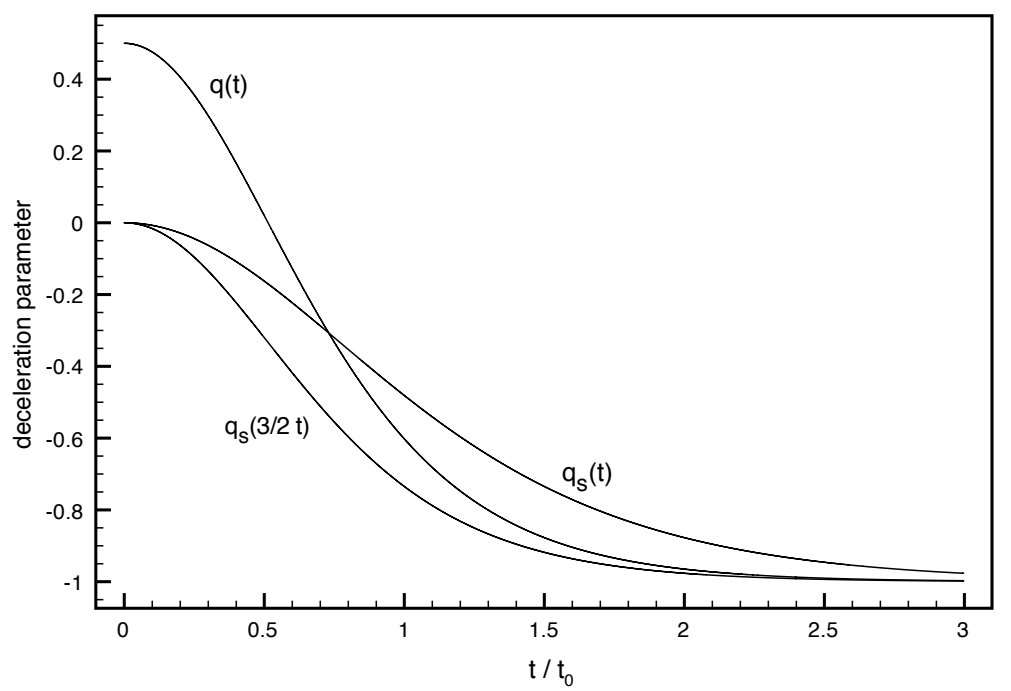

Fig. 3. Deceleration parameters (16) and (32) as functions of time in units of $t_{0}$. The WMAP 7-year data are used (see the caption of Fig. 1)

classical approximation. In terms of general relativity, its effective contribution to the field equations can be linked to the time evolution of the equation of state of matter caused by the processes of redistribution of the energy between its components.

Let us consider the model, in which the equation of state parameter for matter,

$w(t)=\frac{p(t)}{\rho(t)}$,

depends on time ${ }^{1}$.

In the context of the hot Big-Bang cosmology, the radiation-dominated Universe with the energy density $\rho \sim R^{-4}$ transforms in the course of the expansion into the non-relativistic matter-dominated Universe with the energy density $\rho \sim R^{-3}$, and the latter transits to a Universe which looks like that dominated by a perfect gas of low-velocity cosmic strings with $\rho \sim R^{-2}$ at later time. In the radiation-dominated Universe, the number density of photons is $n_{\gamma} \sim R^{-3}$, and the energy of every photon decreases during the expansion of the Universe as $m_{\gamma} \sim R^{-1}$ due to the cosmological redshift. As a result, the effective mass of the Universe attributed to relativistic matter reduces as well, $M_{\text {eff }} \sim m_{\gamma} n_{\gamma} R^{3} \sim R^{-1}$. Arguing in the same way, one finds that, in the matter-dominated Universe, the effective mass is constant, $M_{\mathrm{eff}}=$ const, expressing the constancy of the sum of the masses of bodies in the volume $\sim R^{3}$. In the Universe, which looks like that dominated by a perfect gas of low-velocity cosmic strings,

\footnotetext{
1 Throughout this Section, we assume that $\Lambda=0$ for simplicity.
}

the effective mass of matter increases with the expansion of the Universe, $M_{\text {eff }} \sim R$, due to the redistribution of the energy between the matter components. Thus, we have the following picture of changes of the dominating matter content of the Universe during its evolution in time: the mass of the dominating matter component in the expanding Universe decreases inversely proportional to the "radius" $R$ of the Universe in the radiation-dominated era, then the mass remains constant in the matter-dominated era, and, finally, it increases linearly with $R$, when a gas of point particles (dust) transforms effectively into a perfect gas of lowvelocity cosmic strings. At the same time, the equation of state of the dominating matter changes from the equation $p=\frac{1}{3} \rho$ to $p=-\frac{1}{3} \rho$, by passing through the state $p=0$.

According to the scenario described here, we specify the parameter $w(t)$ in the form of an antikink,

$w(t)=-\frac{1}{3} \tanh \left[\lambda\left(t-t_{0}\right)\right]$

where $t_{0}$ is the instant of time, in the neighborhood of which matter behaves as a pressure-free dust ( $t_{0}$ may be taken close to the age of the Universe), and $\lambda$ is some parameter averaged in time which determines the rate of change of the equation of state.

Parameter (36) can be justified in the model, in which matter in the Universe is described as a two-component perfect fluid with the energy density $\rho=\rho_{1}+\rho_{2}$ and the pressure $p=p_{1}+p_{2}$. We represent the energy conserva- 
tion equation (1) for every component in the form

$\dot{\rho_{1}}+3 H\left(\rho_{1}+p_{1}\right)=Q, \quad \dot{\rho}_{2}+3 H\left(\rho_{2}+p_{2}\right)=-Q$,

where $Q$ describes the interaction between the components.

We assume that the equation of state for the component with the density $\rho_{1}$ changes in time from the stiff Zel'dovich-type equation $p_{1}=\rho_{1}$ to the vacuum-type one $p_{1}=-\rho_{1}$. The second component is a pressure-free matter which has the density $\rho_{2}=2 \rho_{1}$. Then $Q=2 H p_{1}$, and $w=p_{1} /\left(3 \rho_{1}\right)$, while the system of equations (37) reduces to one equation

$\dot{\rho_{1}}+3 H\left(\rho_{1}+\frac{1}{3} p_{1}\right)=0$.

We look for the energy density $\rho_{1}$ in the form

$\rho_{1}=\frac{\alpha(t)}{t^{2}}$,

where $\alpha(t)$ is a slowly varying function on the interval $0<t<\infty$. Then, from the Einstein equation for $\dot{R}$ and Eq. (38) in the approximation $\dot{\alpha} \ll \lambda \alpha$, we find the dependence of the total energy density $\rho$ and the pressure $p$ on time,

$\rho(t)=\frac{3}{2 \pi G t^{2}} \frac{1}{\left[3-\tanh \left(\lambda\left(t-t_{0}\right)\right)\right]^{2}}$,

$p(t)=-\frac{1}{2 \pi G t^{2}} \frac{\tanh \left(\lambda\left(t-t_{0}\right)\right)}{\left[3-\tanh \left(\lambda\left(t-t_{0}\right)\right)\right]^{2}}$.

The Hubble expansion rate is described by the expression

$H(t)=\frac{2}{t} \frac{1}{3-\tanh \left(\lambda\left(t-t_{0}\right)\right)}$.

Equations (40) and (41) reproduce the known expressions for the corresponding quantities in the limiting cases. For $t \rightarrow 0$ and $\lambda t_{0}>2$, we have

$\rho=\frac{3}{32 \pi G t^{2}}, w=\frac{1}{3}, p=\frac{1}{3} \rho, H=\frac{1}{2 t}, R \sim t^{1 / 2}$.

For $t \approx t_{0}$, the expressions are as follows:

$\rho=\frac{1}{6 \pi G t^{2}}, w=0, p=0, H=\frac{2}{3 t}, R \sim t^{2 / 3}$.

While, in the region $t \gg t_{0}$, we have

$\rho=\frac{3}{8 \pi G t^{2}}, w=-\frac{1}{3}, p=-\frac{1}{3} \rho, H=\frac{1}{t}, R \sim t$

(cf. Ref. [34]) and Eq. (20)).
Thus, we have a continuous transition from the era, when radiation dominates over matter, through the era of the dust domination, to the epoch, when matter in the form of low-velocity cosmic strings dominates.

The transition from the radiation-dominated Universe to the Universe dominated by a perfect gas of lowvelocity cosmic strings can be described in terms of a simple string-gas model with the equation of state $p_{s}=w \rho_{s}$, where $w=\frac{2}{3} v_{s}^{2}-\frac{1}{3}$, and $v_{s}$ is the average velocity of cosmic strings [6]. At $v_{s}=1$, the string gas behaves itself as relativistic matter; at $v_{s}=\frac{1}{\sqrt{2}}$, it acts as pressure-free matter; and, at $v_{s}=0$, we have a perfect gas of low-velocity cosmic strings. In such a description, if a gas of low-velocity cosmic strings quickly comes to the domination over relativistic and pressure-free matter, it would drastically alter the cosmological evolution of the Universe. In the model of two-component perfect fluid, this problem is removed, since the era with $\rho \sim R^{-2}$ does not start until the values of the radius and the mass of the observed part of the Universe will become large enough (at least as in the present-day Universe).

1. K. Nakamura et al. (Particle Data Group), J. Phys. G 37, 075021 (2010).

2. A. Riotto, CERN Yellow Report CERN-2010-01, 315 (2010), arXiv:1010.2642 [hep-ph] (2010).

3. E. Bianchi and C. Rovelli, arXiv:1002.3966 [astro-ph.CO] (2010).

4. L. Perivolaropoulos, arXiv:1104.0539 [astro-ph.CO] (2011); L. Perivolaropoulos, in The Problems of Modern Cosmology, edited by P.M. Lavrov (Tomsk State Pedagogical Univ., Tomsk, 2009), arXiv:0811.4684 [astro-ph] (2008); P. Kroupa et al., Astron. Astrophys. 523, A32 (2010), arXiv:1006.1647 [astro-ph.CO] (2010).

5. E.W. Kolb, Astrophys. J. 344, 543 (1989).

6. E.W. Kolb and M.S. Turner, The Early Universe (Addison-Wesley, Redwood City, 1990).

7. G.J. Whitrow and D.G. Randall, MNRAS 111, 455 (1951).

8. D. Meschini, Found. Sci. 12, 277 (2007), arXiv:grqc/0601097 (2006); C. Kiefer and B. Sandhoefer, in Beyond the Big Bang, edited by R. Vaas (Springer, Heidelberg, 2008), arXiv:0804.0672 [gr-qc] (2008).

9. V.V. Kuzmichev, Ukr. J. Phys. 43, 896 (1998); V.V. Kuzmichev, Phys. Atom. Nucl. 62, 708 (1999), arXiv:gr-qc/0002029 (2000); V.V. Kuzmichev, Phys. Atom. Nucl. 62, 1524 (1999), arXiv:gr-qc/0002030 (2000); V.E. Kuzmichev and V.V. Kuzmichev, Eur. Phys. J. C 23, 337 (2002), arXiv:astro-ph/0111438 (2001). 
10. V.E. Kuzmichev and V.V. Kuzmichev, Acta Phys. Pol. B 39, 979 (2008), arXiv:0712.0464 [gr-qc] (2007); V.E. Kuzmichev and V.V. Kuzmichev, Acta Phys. Pol. B 39, 2003 (2008), arXiv:0712.0465 [gr-qc] (2007); V.E. Kuzmichev and V.V. Kuzmichev, Acta Phys. Pol. B 40, 2877 (2009), arXiv:0905.4142 [gr-qc] (2009); V.E. Kuzmichev and V.V. Kuzmichev, Ukr. J. Phys. 55, 626 (2010).

11. F. Lund, Phys. Rev. D 8, 3247 (1973); V.G. Lapchinskii and V.A. Rubakov, Theor. Math. Phys. 33, 1076 (1977); F.J. Tipler, Rep. Prog. Phys. 68, 897 (2005).

12. R.C. Tolman, Relativity, Thermodynamics and Cosmology (Clarendon Press, Oxford, 1969).

13. A.G. Riess et al., Astrophys. J. 730, 119 (2011), arXiv:1103.2976 [astro-ph.CO] (2011).

14. D. Larson et al., Astrophys. J. Suppl. 192, 16 (2011), arXiv:1001.4635 [astro-ph.CO] (2010); N. Jarosik et al., Astrophys. J. Suppl. 192, 14 (2011), arXiv:1001.4744 [astro-ph.CO] (2010).

15. A. Benoit-Levy and G. Chardin, arXiv:0903.2446 [astroph.CO] (2009); arXiv:0811.2149 [astro-ph] (2008).

16. J.R. Gott and M.J. Rees, MNRAS 227, 453 (1987).

17. Ø. Grøn, Eur. J. Phys. 23, 135 (2002), arXiv:0801.0552 [astro-ph] (2008).

18. A. Vilenkin, Phys. Rep. 121, 263 (1985).

19. M. Rowan-Robinson, Cosmology (Clarendon Press, Oxford, 2004).

20. D.W. Sciama, MNRAS 113, 34 (1953).

21. D.W. Sciama, Modern Cosmology (Cambridge Univ. Press, Cambridge, 1971).

22. E. Mach, Die Mechanik in Ihrer Entwickelung: Historisch-Kritisch Dargestellt (F.A. Brockhaus, Leipzig, 1897).

23. H. Bondi and J. Samuel, Phys. Lett. A 228, 121 (1997), arXiv:gr-qc/9607009 (1996).

24. J. Barbour, Found. Phys. 40, 1263 (2010), arXiv:1007.3368 [gr-qc] (2010).

25. J.A. Wheeler, in Gravitation and Relativity, edited by Hong-Yee Chiu and W.F. Hoffmann (Benjamin, New York, 1964).

26. C.H. Brans and R.H. Dicke, Phys. Rev. 124, 925 (1961).

27. M. Heller, Acta Phys. Pol. B 1, 123 (1970).

28. P.A.M. Dirac, Nature 139, 323 (1937).

29. P.A.M. Dirac, Proc. Roy. Soc. London A 333, 403 (1973).

30. S. Weinberg, Gravitation and Cosmology (Wiley, New York, 1972).
31. J.P. Petit, Mod. Phys. Lett. A 3, 1527 (1988); J.W. Moffat, Int. J. Mod. Phys. D 2, 351 (1993), arXiv:grqc/9211020 (1992); A. Albrecht and J. Magueijo, Phys. Rev. D 59, 043516 (1999), arXiv:astro-ph/9811018 (1998).

32. T. Chiba, Prog. Theor. Phys. 126, 993 (2011), arXiv:1111.0092 [gr-qc] (2011).

33. I. Prigogine, J. Geheniau, E. Gunzig, and P. Nardone, Gen. Relativ. Gravit. 21, 767 (1989); J.A.S. Lima, M.O. Calvao, and I. Waga, Cosmology, Thermodynamics and Matter Creation in Frontier Physics, Essays in Honor of Jaime Tiomno, edited by S. MacDowel, H.M. Nussenzweig, and R.A. Salmeron (World Scientific, Singapore, 1990); arXiv:0708.3397 [astro-ph] (2007); A. de Roany and J.A. de Freitas Pacheco, Gen. Relativ. Gravit. 43, 61 (2011).

34. L.D. Landau and E.M. Lifshitz, The Classical Theory of Fields (Butterworth-Heinemann, Amsterdam, 1975).

Received 08.11.11

ПОРІВНЯЛЬНИЙ АНАЛІЗ СТАНДАРТНОЇ $\Lambda$ СDM ТА $\Lambda$ СS МОДЕЛЕЙ

В.С. Кузвмичов, В.В. Кузъмичов

Р е з ю м е

Проведено порівняльний аналіз космологічних параметрів, що залежать від часу та обчислені в рамках стандартної $\Lambda$ CDM моделі, з відповідними параметрами моделі однорідного та ізотропного всесвіту з ненульовою космологічною сталою, який заповнений ідеальним газом космічних струн малої швидкості ( $\Lambda$ CS модель). Показано, що матерія з нульовим тиском може отримати властивості газу космічних струн з малою швидкостю в епоху, коли загальна геометрія та повна кількість матерії у всесвіті як цілому задовольняють додаткове рівняння в'язі. Ця в'язь випливає з квантово-геометродинамічного підходу у квазікласичному наближенні. В рамках загальної теорії відносності її ефективний внесок у польові рівняння може бути пов'язаний з еволюцією у часі рівняння стану матерії, спричиненого процесами перерозподілу енергії між матеріальними компонентами. У даній роботі знайдені точні розв'язки рівнянь Ейнштейна для $\Lambda \mathrm{CS}$ моделі. Показано, що ця модель є еквівалентною відкритій моделі де Сіттера. Після масштабного перетворення часової змінної $\Lambda \mathrm{CS}$ моделі, стандартна $\Lambda \mathrm{CDM}$ та $\Lambda \mathrm{CS}$ моделі забезпечують еквівалентний опис космологічних параметрів як функцій часу при рівних значеннях космологічної сталої. Винятком $є$ поведінка параметра уповільнення у ранньому всесвіті. 